\title{
Effect of modified bipolar tonsillectomy on postoperative pain
}

\author{
Gevorg Saghatelyan ${ }^{1 *}$ and Vahan Nalbandyan ${ }^{2}$ \\ ${ }^{1}$ Arabkir Medical Centre, Department of Otorhinolaryngology, Yerevan, Armenia \\ ${ }^{2}$ Astghik Medical Centre (AMC), Department of Otorhinolaryngology, Yerevan, Armenia
}

\begin{abstract}
Background: Tonsillectomy is commonly performed otolaryngological operation. It can cause considerable pain in children. Different types of tonsillectomy are described in the literature. Modified technique of bipolar tonsillectomy can significantly reduce postoperative pain.

Objective: This study aimed to assess postoperative pain in children after modified bipolar tonsillectomy.

Methods: 120 patients, age 6-12 years, were selected for this study. Indication for tonsillectomy was a history of recurrent tonsillitis. Spetzler-Malis bipolar forceps (tip size $0.5 \mathrm{~mm}$ ) with low energy $(5 \mathrm{~W})$ was applied. We tried to preserve the pillars as much as possible. Pain was assessed on $1 \mathrm{st}$, $3 \mathrm{rd}$, 5 th, 7 th and 10 th postoperative days. The pain was evaluated with visual analogue scale (VAS).

Results: On the first day of the operation 114 patients (95\%) had no pain, 6 patients (5\%) had mild pain, on the third postoperative day in $95 \%$ pain was mild, in $5 \%$ pain was moderate, on the fifth postoperative day pain was mild in 84 patients (70\%), 36 patients (30\%) had moderate pain, on the seventh postoperative day $95 \%$ of patients did not present pain, $5 \%$ of patients had mild pain. On the tenth postoperative day no one had pain. None of them presented nausea, vomiting, otalgia or severe pain.
\end{abstract}

Conclusion: Low energy electrocautery, direct cautery to the tonsillar bed and preservation of the mucosa are all effective measures that have diminished post-op pain in bipolar dissection.

\section{Introduction}

Tonsillectomy is one of the most common surgeries performed in pediatric population. Unlike most operative procedures, which are closed primarily, tonsillectomy produces an open wound that heals by secondary intention. The major postoperative morbidity problems are pain and delayed hemorrhage. The pain is the result of disruption of mucosa and glossopharyngeal and/or vagal nerve fibers followed by inflammation and spasm of the pharyngeal muscles that leads to ischemia and a protracted cycle of pain [1-9].

These complications lead to the development of different techniques. Tonsillectomy via cold dissection is a traditional technique and newly introduced techniques include bipolar cautery, coblation, harmonic scalpel, powered intracapsular tonsillectomy [2]. Despite the different techniques available for tonsillectomy, there is no consensus and conclusive evidence in the literature to date on the optimum or the best method of performing the tonsillectomy, and none of the techniques has been accepted as the best one universally [1,5-12].

Some investigations believe that hot methods damage tissues and create burning wounds that can lead to increased postoperative pain as well as delayed epithelialization in the surgical bed. Another group of otorhinolaryngologists prefers thermal methods. They suggest that bipolar instruments (forceps and scissors) cause less thermal damage and less postoperative pain compared with monopolar electrocautery, but reports have demonstrated a higher incidence of postoperative pain and bleeding with these instruments compared with tonsillectomy performed by cold dissection with the scalpel $[2,8,12]$. Some of the studies came to the conclusion that diathermy increases postoperative pain and bleeding [3].

In our experience, we can conclude that using a modified technique with lower thermal trauma will significantly reduce postoperative pain.

\section{Materials and methods}

Pediatric patients $(n=120)$ between age group of 6-12 years (mean age 7.42, SD 3.69) were selected for tonsillectomy. Indication for tonsillectomy was a history of recurrent tonsillitis. Exclusion criteria included patients with bleeding disorders, previous history of peritonsillar abscess, any major illness or medical problems, contraindication to general anesthesia, contraindication to paracetamol, craniofacial anomalies and difficult contact or communication with the parents.

No topical or local anesthesia, antibiotics or other medications were administered before surgery.

All procedures were performed under general anesthesia through endotracheal intubation and the anesthesia protocols were standard for all patients.

${ }^{*}$ Correspondence to: Gevorg Saghatelyan, Arabkir Medical Centre, Department of Otorhinolaryngology, Yerevan, Armenia, Tel: +37498118619, E-mail: ruslangevorg@gmail.com

Key words: bipolar, children, pain, tonsillectomy

Received: January 11, 2021; Accepted: January 26, 2021; Published: January 29 2021 
The patients were placed in the Rose position and a McIvor mouth gag was inserted into the mouth. Headlight was used for illumination. Spetzler-Malis bipolar forceps (tip size $0.5 \mathrm{~mm}$ ) was applied (Figure 1). Low energy bipolar cautery technique of $5 \mathrm{~W}$ was used to reduce heat trauma to the tonsillar bed. The tonsil was grasped near its superior pole and drawn medially. A classical mucosal incision is not performed in our technique (Figure 2). We made an incision at the mucotonsillar transition with bipolar forceps (Figure 3). Also, we used the bipolar forceps as a dissector. Small vessels were detected, and cautery was performed before sectioning. Early identification decreases the need for aggressive cautery. When the peritonsillar loose connective tissue was identified, the tonsil was completely removed. We did not use tonsil snare. Any further hemostasis of the tonsillar fossa was secured with bipolar forceps. Tonsillar fossa was hardly visible or not visible after removing tonsils (Figure 4). We retracted the anterior pillar with elevator for inspection of the tonsillar fossa (Figure 5).

All patients were discharged the day after surgery and then reviewed on 3rd, 7th, and 10th post-op days. Analgesic drug (acetaminophen) was administrated as $15 \mathrm{mg} / \mathrm{kg}$ if required.

Pain was assessed on 1st, 3rd, 5th, 7th, and 10th postoperative days. Pain was evaluated using visual analogue scale (VAS) on a scale of $0-10$, with 0 representing no pain at all and 10 the worst possible pain (Figure 6). VAS scores 4-6 were considered as moderate. Self-assessment scales are only reliable after age 6 years because they require cognitive and language skills. Self-reporting using the visual analogue scale (VAS) is the gold standard and is the most validated tool [13-24]. Before surgery, the child and parents were instructed on the use of the pain scale. They were also asked to report the amount of analgesic that the children needed to take.

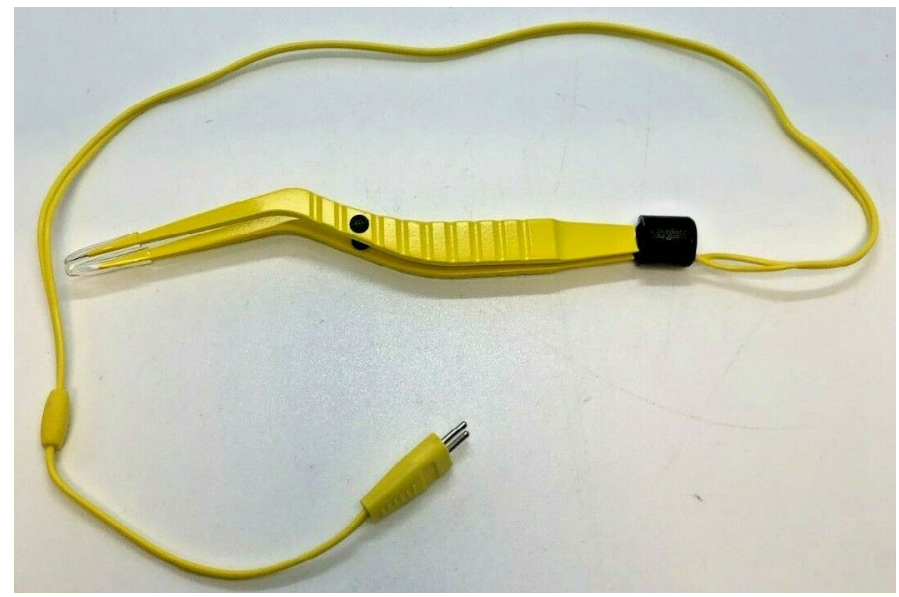

Figure 1. Spetzler-Malis bipolar forceps

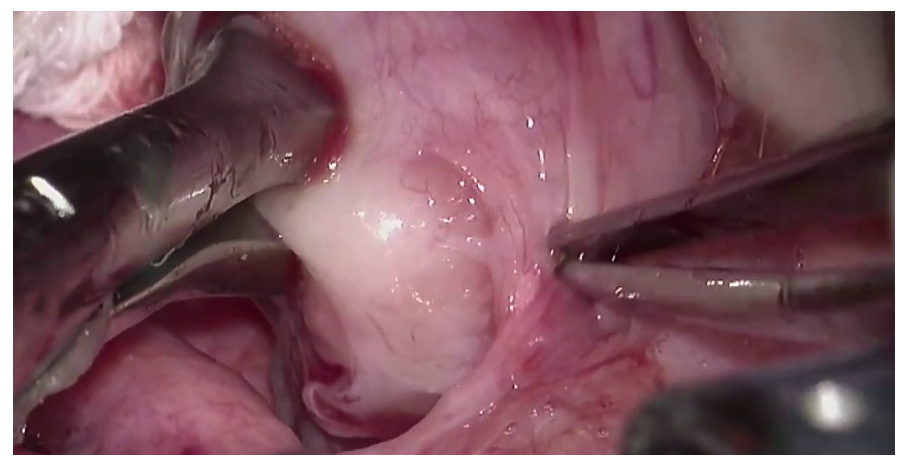

Figure 2. Classical incision site is shown with the instrument

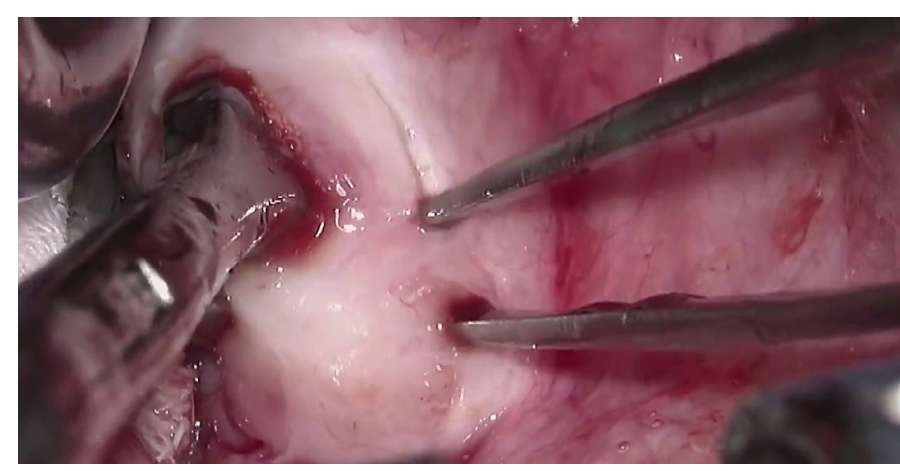

Figure 3. With the instrument is shown modified incision site (at the mucotonsillar transition)

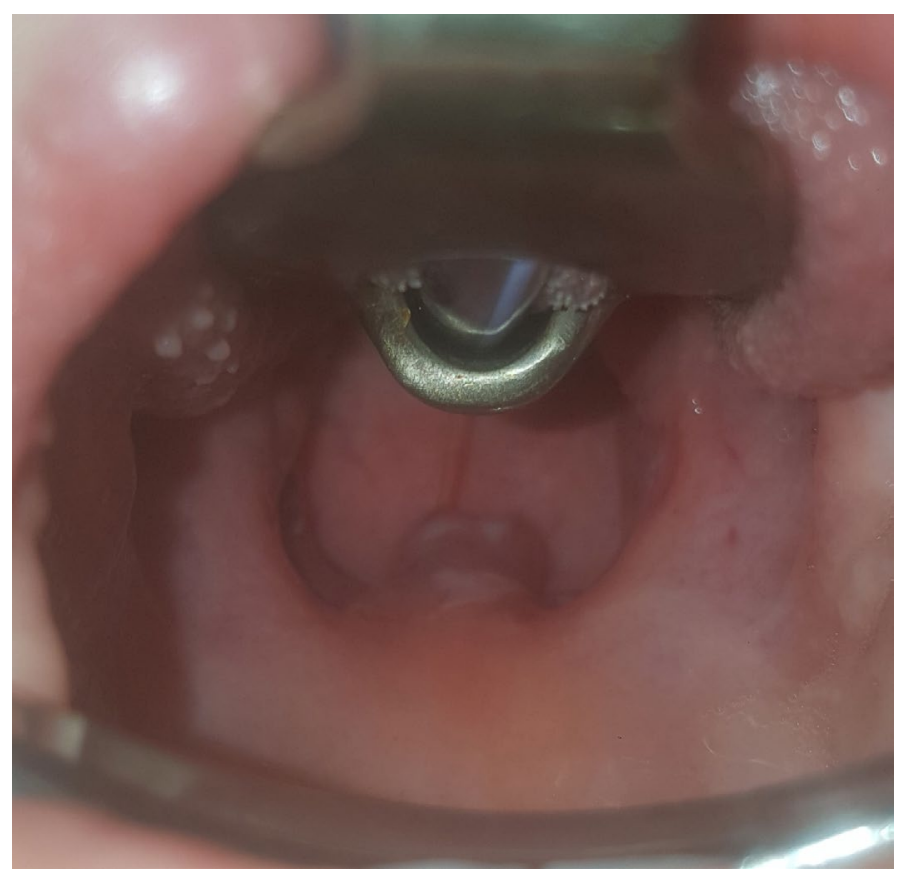

Figure 4. Postoperative view. Tonsillar fossa is hardly visible or not visible. The pillars are not sacrificed

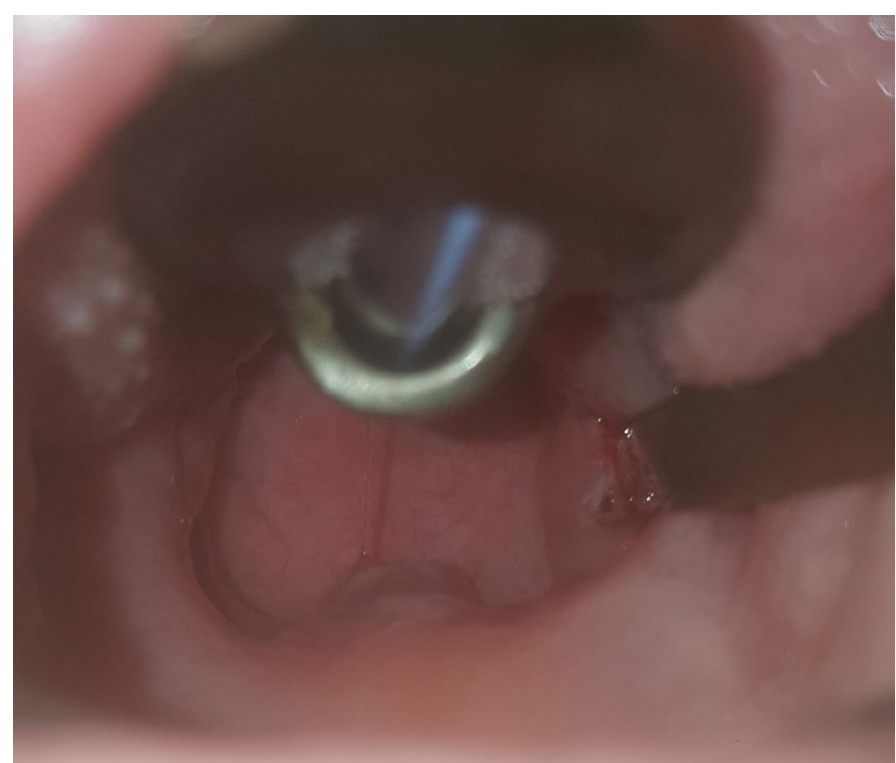

Figure 5. The right anterior pillar is retracted with elevator to make tonsillar fossa visible 

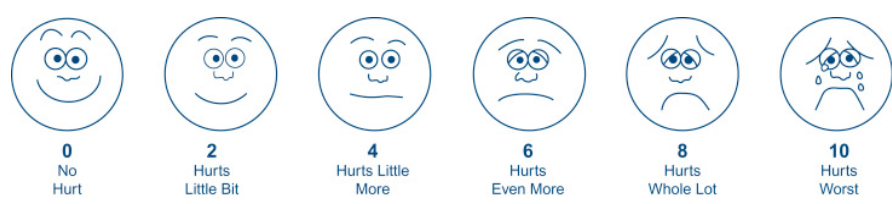

Figure 6. Visual analogue scale (VAS).

Table 1. Mean pain scores

\begin{tabular}{|l|c|c|c|c|}
\hline & Day 1 & Day 3 & Day 5 & Day 7 \\
\hline Mean VAS** score & 0.1 & 2.1 & 2.7 & 0.1 \\
\hline SD $\dagger$ & 0.191 & 0.191 & 1.32 & 0.191 \\
\hline SE $\dagger \dagger$ & 0.0016 & 0.0016 & 0.011 & 0.0016 \\
\hline
\end{tabular}

$* *$ Visual analogue scale, $\uparrow$ standard deviation, $\dagger \dagger$ standard error

\section{Results}

On the first day of the operation 114 patients (95\%) had no pain, 6 patients (5\%) had mild pain (mean VAS score 0.1, range 0-2, SD 0.191, SE 0.0016 ), on the third postoperative day in $95 \%$ pain was mild, in $5 \%$ pain was moderate (mean VAS score 2.1 , range $2-4$, SD $0.191, \mathrm{SE}$ $0.0016)$, on the fifth postoperative day pain was mild in 84 patients (70\%), 36 patients $(30 \%)$ had moderate pain (mean VAS score 2.7 , range $2-6$, SD 1.32, SE 0.011 ), on the seventh postoperative day $95 \%$ of patients did not present pain, $5 \%$ of patients had mild pain (mean VAS score 0.1 , range $0-2$, SD 0.191, SE 0.0016). On the tenth postoperative day no one had pain. Mean pain scores are shown in Table 1.

The patients did not present nausea, vomiting, otalgia or severe pain during postoperative days. Only patients with moderate pain needed analgesic. Mean consumption of analgesics on the third day was 1 tablet of acetaminophen $500 \mathrm{mg}$ (SD 0.96, SE 0.008), on the fifth day was 1.17 tablet (SD 1.008, SE 0.0084).

\section{Discussion}

Tonsillectomy is one of the most frequently performed otorhinolaryngological procedures, accounting for about $20 \%$ of all operations [4]. The morbidity of this operation may be significant, so surgeons must consider haemorrhage, apnea, pain, fever and poor oral intake as possible effects of the surgery. Various surgical techniques were developed to achieve less blood loss and pain after tonsillectomy [19]. The cold dissection tonsillectomy is a traditional technique of surgery, meanwhile, monopolar electrocautery tonsillectomy is a preferred technique, but it is associated with more pain and slower healing $[2,20]$. Bipolar electrocautery is preferred over monopolar cautery for tonsillectomy because the spread of heat is less and more controlled and consequently the tissue damage and postoperative pain are reduced [3,21-40]. Several studies support the hypothesis that the extent of diathermy used in tonsillectomy has a direct influence on the delayed postoperative morbidity and healing of the mucosal wounds [1]. Microdebrider, coblation, radiofrequency and laser are other surgical techniques used for tonsillectomy [7]. Surgical technique can also be modified to decrease pain; intracapsular tonsillectomy has been shown to reduce the severity and duration of pain. Cold dissection techniques lead to less pain, but hot techniques remain popular, with less intraoperative blood loss and shorter operative time [25].

Ideal tonsillectomy should be quick, painless, and associated with no blood loss. However, to date none of the commonly used techniques has been shown to be superior. Attempts are made to find an ideal surgical method with shorter operative time, less intraoperative blood loss, quick recovery, and less pain [15,26-38]. Despite the frequency of tonsillectomy, not universally accepted "ideal" method is discovered yet
[39]. Painless tonsillectomy is still a challenge that is as desirable as it is utopia-like [13].

Post-op pain is one of the most vexing side effects and is reportedly the most common cause for patients' referral during the first two weeks of post-op visits [7,22]. Pain is usually caused by heat or mechanical trauma to adjacent tissues that is eventually accompanied by edema, pharyngeal muscles spasm, and tonsillar bed nerve endings irritation. Regarding the annoying nature of post-op pain, many studies have been developed to compare the pain severity of different surgical techniques. Although bipolar electrocautery dissection has less intraoperative bleeding, controversial findings have been reported regarding its postop pain severity and patient comfort [7].

The reduction of postoperative pain is important, not only for patient comfort, but also because reducing pain improves oral intake, which in turn decreases the risk of dehydration, infection and, thus, postoperative complications such as delayed hemorrhage $[1,11,21]$.

Numerous studies in the literature suggest that temperature is the most important factor in preventing tissue damage and postoperative pain [8]. Evidence suggests that none of the hot tonsillectomy techniques offers concurrent reductions in intra- and post-operative bleeding and pain, compared with traditional cold-steel dissection with packs or ties [6]. Bipolar electrodissection tonsillectomy (BET) produces more post-operative pain and a delayed return to normal diet, compared with cold dissection tonsillectomy (CDT). The current data support previous evidence that electrodissection tonsillectomy increases postoperative pain after hospital discharge [12,16]. In Atallah [3] study pain was much less with the dissection ligation technique compared to the electrodissection method. In a systematic review of Leinbach et al. [26] electrodissection caused increased postoperative pain in comparison with cold dissection tonsillectomy. Cardozo et al. [27] also found a significant relationship between bipolar diathermy energy and postoperative pain in adult tonsillectomy. In the study of Salehi at al. [5] the patients undergoing tonsillectomy with the BET experienced higher pain intensity than the CDT group. Similarly, higher pain intensity scores with BET have been reported by Gendy et al. [30]. In Ali et al's [31] study, although, the initial postoperative pain was not different statistically between BET and CDT groups, later, on 7th and 14th days after surgery, the severity of pain was significantly higher in patients who underwent tonsillectomy with BET. In studies carried out by Chettri et al. [32,33], Adoga [34] and Bukhari et al. [35] a significantly greater percentage of patients in the BET group complained of higher pain intensity than the CDT group. Vithayathil et al. [36] and Hashemi et al. [37] showed no significant difference between two studied groups BET and CDT [39,40]. In Ozkiris [41] study, the post-op pain score was also reported to be higher in the electrocautery dissection technique than in the cold dissection. Conversely, Raut et al. [42] showed that the post-op pain score in the electrocautery dissection tonsillectomy technique was not statistically different with cold dissection. They claimed that the electrocautery dissection was an ideal method for pediatric tonsillectomy due to lessened bleeding and a shorter operation time. Nunez et al. [28] using number of postoperative analgesic doses as a measure of pain, showed that children who underwent tonsillectomy with electrocauetry took 7.5 more doses of analgesics than those undergoing cold dissection. They reported that children in the bipolar diathermy group took 2.5 days more to return to normal diet. Pang [29] reported that children in the bipolar diathermy group were able to drink and eat significantly earlier than those in the dissection/snare group. In Leaper's [14] study results suggest there is no advantage with the use of the harmonic scalpel over the bipolar diathermy with regards 
to operating time, post-operative morbidity, and bleeding for pediatric tonsillectomy. Singh et al. [18] concluded that postoperative pain was less with coblator assisted tonsillectomy as compared to bipolar diathermy tonsillectomy at least in early postoperative period.

The observed differences of pain intensity in different studies could possibly be related to various factors such as length of disease, amount and severity of employed energy during surgery, the generated heat leading to tissue burning, size of the cut area, ability to tolerate pain etc.

The present study was conducted to evaluate postoperative pain with modified bipolar tonsillectomy technique. For the pain assessment in the present study, the visual analogue scale (VAS) was used. In addition, the number of analgesics was recorded as a surrogate measure of pain. The VAS has been used previously for the assessment of postoperative pain in adults and children following tonsillectomy [10].

We found that during first day after surgery $95 \%$ of patients had no pain, $5 \%$ of patients had mild pain, during third postoperative day $95 \%$ of patients had mild pain, $5 \%$ of patients had moderate pain, on the fifth postoperative day pain was mild in $70 \%$ of patients, $30 \%$ of patients had moderate pain, on the seventh postoperative day $95 \%$ of patients did not present pain, $5 \%$ of patients had mild pain. On the tenth postoperative day none of them had pain. Otalgia, severe pain, nausea and vomiting were not presented during postoperative period.

The key was maximum preservation of the mucosa and minimum damage to the tissues. Post-tonsillectomy pain and bleeding risk generally continue until the tonsillar fossae are fully mucosalized. Epithelial ingrowth from the cut edges of the anterior and posterior tonsillar pillars provides much of this remucosalization. Large tissue defects and thermal damage to the pillar mucosa can thus delay healing and increase the potential for discomfort and late complications. This has great appeal to any surgeon. Unfortunately, more of the anterior pillar is sacrificed by using some techniques.

\section{Conclusion}

Although we did not make comparisons between different techniques, we have observed that modified bipolar technique can significantly reduce postoperative pain. Low energy electrocautery, direct cautery to the tonsillar bed and preservation of the mucosa are all effective measures that have diminished post-op pain in bipolar electrocautery dissection.

\section{References}

1. Bäck L, Paloheimo M, Ylikoski J (2001) Traditional tonsillectomy compared with bipolar radiofrequency thermal ablation tonsillectomy in adults. Arch Otolaryngol Head Neck Surg 127: 1106-1112.

2. Dadgarnia MH, Ali MA, Atighechi S, Behniafard N, Reza MV, et al. (2016) The comparison of bleeding and pain after tonsillectomy in bipolar electrocautery vs cold dissection. Int J Pediatr Otorhinolaryngol 89: 38-41. [Crossref]

3. Atallah N, Kumar M, Hilali A, Hickey S (2000) Post-operative pain in tonsillectomy: bipolar electrodissection technique vs dissection ligation technique. A double-blind randomized prospective trial. J Laryngol Otol 114: 667-670. [Crossref]

4. Palacios IA, Martınez CA, Mejıas AA, Martínez CA, Mejías AA, et al. (2017) Postoperative pain in adult tonsillectomy: is there any difference between the technique? Indian J Otolaryngol Head Neck Surg 69: 187-193. [Crossref]

5. Salehi F, Mofatteh MR, Hosseini M, Hassanzadeh-Taheri M, Sharifzadeh G, et al. (2020) Comparison of postoperative morbidity between conventional cold dissection and bipolar electrocautery tonsillectomy: which technique is better? $\mathrm{Br} J$ Otorhinolaryngol 86: 427-433.

6. Scott A (2006) Hot techniques for tonsillectomy. Issues Emerg Health Technologies 93: 1-6.
7. Bagherihagh A, Sadr Hosseini SM (2017) Two Techniques of Tonsillectomy Performed in Identical Twins: a case report. Iran J Otorhinolaryngol 29: 43-46. [Crossref]

8. Vieira L, Nissen L, Sela G, Amara Y, Fonseca V (2014) Reducing postoperative pain from tonsillectomy using monopolar electrocautery by cooling the oropharynx. Int Arch Otorhinolaryngol 18: 155-158.

9. Hesham A (2009) Bipolar diathermy versus cold dissection in paediatric tonsillectomy. Int J Pediatr Otorhinolaryngol 73: 793-795.

10. Arbin L, Enlund M, Knutsson J (2017) Post-tonsillectomy pain after using bipolar diathermy scissors or the harmonic scalpel: a randomised blinded study. Eur Arch Otorhinolaryngol 274: 2281-2285.

11. Kirazli T, Bilgen C, Midilli R, Meltem Uyar FÖ, Kedek A (2005) Bipolar electrodissection tonsillectomy in children. Eur Arch Otorhinolaryngol 262: 716-718. [Crossref]

12. Mofatteh MR, Salehi F, Hosseini M, Hassanzadeh-Taheri M, Meghdadi S, et al. (2019) Postoperative outcomes in cold dissection versus bipolar electrocautery tonsillectomy: a randomized double-blind controlled study. Indian J Otolaryngol Head Neck Surg 71: 182-187. [Crossref]

13. García Callejo FJ, Piedrahita IR, Gandía RM, Valenzuela OS, Beneyto MPM, et al. (2016) Factors related to post-tonsillectomy pain in adults. Acta Otorrinolaringologica 67: 23-32. [Crossref]

14. Leaper M, Mahadevan M, Vokes D, Sandow D, Anderson BJ, et al. (2006) A prospective randomised single blinded study comparing harmonic scalpel tonsillectomy with bipolar tonsillectomy. Int J Pediatr Otorhinolaryngol 70: 1389-1396.

15. Isaacson G, Szeremeta W (1998) Pediatric tonsillectomy with bipolar electrosurgica scissors. Am J Otolatyngol 19: 291-295. [Crossref]

16. Ragab SM (2012) Six years of evidence-based adult dissection tonsillectomy with ultrasonic scalpel, bipolar electrocautery, bipolar radiofrequency or 'cold steel' dissection. J Laryngol Otol 126: 1056-1062. [Crossref]

17. Andrea M (1993) Microsurgical bipolar cautery tonsillectomy. Laryngoscope 103 1177-1178.

18. Bhardwaj B, Singh J (2018) Comparative post-operative pain analysis between coblator assisted and bipolar diathermy tonsillectomy in paediatric patients. Indian J Otolaryngol Head Neck Surg 71: 90-94.

19. De S (2017) The Airway. Pediatric otolaryngology: practical clinical management Thieme Publishers. Stuttgart. pp: 5.

20. Bajaj Y, Hore I (2018) Diseases of tonsils, tonsillectomy and tonsillotomy. ScottBrown's otorhinolaryngology head and neck surgery. CRC Press. Boca Raton. pp: 38.

21. Cunningham MJ, Myers MC III (2005) Tonsillectomy and adenoidectomy Complications in pediatric otolaryngology. CRC Press. Boca Raton. pp: 15.

22. Ow TJ, Parikh SR (2009) Complications of tonsillectomy and adenoidectomy. Complications in head and neck surgery. Elsevier, Philadelphia. pp: 28.

23. Fink R (2000) Pain assessment: the cornerstone to optimal pain management. Proc (Bayl Univ Med Cent) 13: 236-239.

24. Beltramini A, Milojevic K, Pateron D (2017) Pain assessment in newborns, infants and children. Pediatric Annals 46 (10): 387-395.

25. Tan GX, Tunkel DE (2017) Control of pain after tonsillectomy in children: a review. JAMA Otolaryngol Head Neck Surg 143: 937-942. [Crossref]

26. Leinbach RF, Markwell SJ, Colliver JA, Lin SY (2003) Hot versus cold tonsillectomy: a systematic review of the literature. Otolaryngol Head Neck Surg 129: 360-364. [Crossref]

27. Cardozo AA, Hallikeri C, Lawrence H, Sankar V, Hargreaves S (2007) Teenage and adult tonsillectomy: dose-response relationship between diathermy energy used and morbidity. Clin Otolaryngol 32: 366-371.

28. Nunez DA, Provan J, Crawford M (2000) Postoperative tonsillectomy pain in pediatric patients. Arch Otolaryngol Head Neck Surg 126: 837-841.

29. Pang YT (1995) Paediatric tonsillectomy: bipolar electrodissection and dissection snare compared. J Laryngol Otol 109: 733-736. [Crossref]

30. Gendy S, O'Leary M, Colreavy M, Rowley H, O’Dwyer T, Blayney A (2004) Tonsillectomy cold dissection vs. hot dissection: a prospective study. Ir Med J 98: 243-244. [Crossref]

31. Ali M, Rafique A, Dastgir M, Rashid M, Maqbool S, et al. (2014) Comparison of bipolar electrocautry and cold steel dissection methods for tonsillectomy. PAFM J 64: 34-38. 
32. Manandhar S, Bhandary S, Chhetri ST, Khanal B, Shah S, et al. (2016) Bacteriological evaluation of tonsillar surface and tonsillar core micro flora in patients undergoing tonsillectomy. Health Renaiss 12: 149-153.

33. Chettri ST, Bhandary S, Nepal A, Joshi RR, Natesh V, et al. (2014) A single blind controlled study comparing bipolar elecrocautery tonsillectomy to cold dissection method in pediatric age groups. Health Renaiss 11:270-272.

34. Adoga A (2011) Cold versus hot dissection tonsillectomies: the Nigerian experience. ASEA 16: 64-68

35. Bukhari MA, Al-Ammar AY (2007) Monopolar electrodissection versus cold dissection tonsillectomy among children. Saudi Med J 28: 1525-1528. [Crossref]

36. Vithayathil AA, Maruvala S (2017) Comparison between cold dissection snare method and bipolar electrodissection method in tonsillectomy. Res Otolaryngol 6: 17-22.
37. Hashemi M, Salmani A, Abtahi M (2002) Post tonsilectomy morbidities: bipolar electrosurgical scissor vs classic method. JRMS 7: 324-327.

38. Leach J, Manning S, Schaefer S (1993) Comparison of two methods of tonsillectomy. Laryngoscope 103: 619-622. [Crossref]

39. Pinder DK, Wilson H, Hilton MP (2011) Dissection versus diathermy for tonsillectomy Cochrane Database Syst Rev 3: Cd002211. [Crossref]

40. Kousha A, Banan R, Fotoohi N, Banan R (2007) Cold dissection versus bipolar electrocautery tonsillectomy. J Res Med Sci 12: 117-120. [Crossref]

41. Özkırıs M (2012) Comparison of three techniques in pediatric tonsillectomy. Eur Arch Otorhinolaryngol 269: 1497-1501. [Crossref]

42. Raut VV, Bhat N, Sinnathuray AR, Kinsella JB, Stevenson M, et al. (2002) Bipola scissors versus cold dissection for pediatric tonsillectomy-a prospective: randomized pilot study. Int J Pediatr Otorhinolaryngol 64: 9-15. [Crossref]

Copyright: $\left(\mathbb{C}^{2021}\right.$ Saghatelyan G. This is an open-access article distributed under the terms of the Creative Commons Attribution License, which permits unrestricted use, distribution, and reproduction in any medium, provided the original author and source are credited. 